\title{
ON APPROXIMATION TO AN ANALYTIC FUNCTION BY POLYNOMIALS*
}

BY O. J. FARRELL

1. Introduction. If the function $f(z)$ is analytic in the interior $R$ of a Jordan curve $C$ and continuous in the corresponding closed region $\bar{R}$, then in that closed region $f(z)$ can be uniformly expanded in a series of polynomials in $z$. This result is due to $W a l s h, \dagger$ who recently encouraged the writer to study the problem of polynomial approximation to a function $f(z)$ analytic interior to $C$ but not necessarily continuous in $\bar{R}$ or even bounded in $R$. Professor Walsh suggested (1) that if $f(z)$ is bounded in $R$, there might exist polynomials $p_{n}(z),(n=1,2$, ‥), which approximate to $f(z)$ in $R$ in such a way that the limit as $n \rightarrow \infty$ of the least upper bound of $\left|p_{n}(z)\right|$ for $z$ in $R$ does not exceed the least upper bound of $|f(z)|$ for $z$ in $R$; (2) that if the double integral over $R$ of $|f(z)|^{p},(p>0)$, exists, there might be a sequence of polynomials $\left\{p_{n}(z)\right\}$ which approximate to $f(z)$ in $R$ so that the limit as $n \rightarrow \infty$ of the double integral over $R$ of $\left|f(z)-p_{n}(z)\right|^{p}$ is zero. It is the purpose of the present note to show that the existence of such approximating polynomials can be established in both cases by a method due to Carleman $\ddagger$ not only for a Jordan region but also for regions of somewhat more general boundary.

It is a well known characteristic of a finite simply connected region $R$ bounded by a Jordan curve $C$ that the totality of points of the extended plane not belonging to $R+C$ form a single region (also simply connected) whose boundary is precisely $C$. But the class of regions characterised by this property includes not only all finite Jordan regions but regions of more general boundary as well. $\S$ And it has been found that the results sug-

\footnotetext{
* Presented to the Society, March 31, 1934.

$\dagger$ Walsh, Mathematische Annalen, vol. 69 (1926), p. 430.

¥ Carleman, Arkiv för Matematik, Astronomi och Fysik, vol. 17 (1923), pp. $1-30, \S 1$.

$\S$ For an example of such a region with a boundary which is not a Jordan curve see, for instance, Carathéodory, Mathematische Annalen, vol. 73, pp. $305-370, \S 35$.
} 
gested by Walsh hold for an arbitrary region of this class. More precisely stated the results obtained are given by the following two theorems.

THEOREM 1. In the z-plane let $G$ be a finite simply connected region whose boundary $B$ is such that all the points of the extended plane not on $G+B$ form a single region whose boundary is precisely $B$. If the function $f(z)$ is analytic and bounded in $G$, then there exist polynomials $p_{n}(z),(n=1,2, \cdots)$, which converge to $f(z)$ continuously* in $G$ as $n \rightarrow \infty$ and for which

(1) $\left\{\lim _{n \rightarrow \infty}\left\{\overline{\text { bound }}\left[\left|p_{n}(z)\right|, z\right.\right.\right.$ in $\left.\left.G\right]\right\} \leqq \overline{\text { bound }}[|f(z)|, z$ in $G]$.

Theorem 2. In the z-plane let $G$ be a finite simply connected region of the kind described in Theorem 1. If the function $f(z)$ is analytic in $G$ and such that

$$
\iint_{G}|f(z)|^{p} d S, \quad(0<p),
$$

exists, then there exist polynomials $p_{n}(z),(n=1,2, \cdots)$, which converge to $f(z)$ continuously in $G$ as $n \rightarrow \infty$ and such that

$$
\lim _{n \rightarrow \infty} \iint_{G}\left|f(z)-p_{n}(z)\right|^{p} d S=0 .
$$

In a subsequent paper the writer hopes to tell what is the most general finite simply connected region to which each of these two theorems can be extended.

2. Proof of Theorem 1. Let $G_{n},(n=1,2, \cdots)$, be a set of uniformly bounded simply connected regions such that the closed region $\bar{G}=G+B$ lies in every $G_{n}$ and the region $G$ is the kernel $\dagger$ of every infinite subsequence of the sequence $\left\{G_{n}\right\}$. Let the function $\psi_{n}(z)$ map the region $G_{n}$ conformally onto the region $G$ in such a way that a fixed point $z_{0}$ in $G$ and a fixed direction at $z_{0}$ remain invariant. It follows then from results of Bieberbach $\ddagger$ that

\footnotetext{
* See Walsh, Transactions of this Society, vol. 33 (1931), pp. 668-689, p.669.

$\dagger$ See Carathéodory, Mathematische Annalen, vol. 72 (1912), pp. 107-144 p. 124, or Bieberbach, Lehrbuch der Funktionentheorie, vol. 2, 1927, pp.12-13.

$\ddagger$ Bieberbach, loc. cit., p. 13.
} 


$$
\lim _{n \rightarrow \infty} \psi_{n}(z)=z, \quad \lim _{n \rightarrow \infty} \psi_{n}^{\prime}(z)=1
$$

continuously in $G$. Hence the functions

$$
f_{n}(z)=f\left[\psi_{n}(z)\right], \quad(n=1,2, \cdots),
$$

are analytic in the closed region $\bar{G}$ and they converge to $f(z)$ continuously in $G$. Moreover, it is to be noted that

$$
\overline{\text { bound }}\left[\left|f_{n}(z)\right|, z \text { in } G\right] \leqq \overline{\text { bound }}[|f(z)|, z \text { in } G]
$$

for every $n$, since the set of values which $f_{n}(z)$ takes on in $G$ is precisely the set of values which $f(z)$ takes on in the transform of $G$ by the mapping relation $\psi_{n}(z)$, and this transform of $G$ lies interior to $G$.

By a theorem of Walsh* the function $f_{n}(z)$ can be uniformly approximated as closely as desired in $\bar{G}$ by a polynomial in $z$. If we choose polynomials $\left\{p_{n}(z)\right\}$ so that $\left|f_{n}(z)-p_{n}(z)\right| \leqq \epsilon_{n}$ for $z$ in $\bar{G}$, where $\lim _{n \rightarrow \infty} \epsilon_{n}=0$, we have at once a set of polynomials $\left\{p_{n}(z)\right\}$ which converge to $f(z)$ continuously in $G$ and which satisfy (1).

We have not used here the fact that the derivative of $\psi_{n}(z)$ converges to unity continuously in $G$ as $n \rightarrow \infty$. This fact will, however, be required in the proof of Theorem 2 .

3. Proof of Theorem 2. We require for later application in the proof of this theorem the following lemma on double integrals.

LEMMA. If the functions $f(z)$ and $\phi(z)$ are continuous in a region $R$ of the z-plane and such that

$$
\iint_{R}|f(z)|^{p} d S=M, \quad \iint_{R}|\phi(z)|^{p} d S=N, \quad(0<p),
$$

then

$$
\iint_{R}|f(z)-\phi(z)|^{p} d S \leqq 2^{p}(M+N)
$$

*Walsh, loc. cit., bottom of page 433 and top of page 434 . 
To prove this lemma consider the real continuous non-negative function $g(z)$ which is equal to $|f(z)|$ when $|f(z)|=|\phi(z)|$ and is equal to the greater of these two absolute values when they are unequal. It follows at once from the definition of $g(z)$ that the double integral over $R$ of $[g(z)]^{p}$ exists and that

$$
\iint_{R}[g(z)]^{p} d S \leqq M+N .
$$

But since $|f(z)-\phi(z)| \leqq|f(z)|+|\phi(z)| \leqq 2 g(z)$, it is clear that (3) follows from (4), and the lemma is proved.

Let $\left\{G_{n}\right\}$ and $\left\{\psi_{n}(z)\right\}$ denote respectively the regions used in the proof of Theorem 1 and their mapping functions. Let $\Gamma_{n}$ denote the region into which $G$ is transformed by $\psi_{n}(z)$. If the proper determination of each of the functions

$$
F_{n}(z)=f\left[\psi_{n}(z)\right]\left[\psi_{n}^{\prime}(z)\right]^{2 / p}, \quad(n=1,2, \cdots),
$$

be chosen (and we will suppose that this has been done), then the functions $F_{n}(z)$ are analytic everywhere in the closed region $\bar{G}$ and $\lim _{n \rightarrow \infty} F_{n}(z)=f(z)$ continuously in $G$. Moreover

$\lim _{n \rightarrow \infty} \iint_{G}\left|F_{n}(z)\right|^{p} d S=\lim _{n \rightarrow \infty} \iint_{\Gamma_{n}}|f(z)|^{p} d S=\iint_{G}|f(z)|^{p} d S$.

But by the theorem of Walsh used in the proof of Theorem 1 each function $F_{n}(z)$ can be uniformly approximated as closely as desired in $\bar{G}$ by a polynomial in $z$. So it is clear that there exist polynomials $\left\{p_{n}(z)\right\}$ which converge to $f(z)$ continuously in $G$ and for which

$$
\lim _{n \rightarrow \infty} \iint_{G}\left|p_{n}(z)\right|^{p} d S=\iint_{\dot{G}}|f(z)|^{p} d S .
$$

It remains to show that these polynomials also satisfy (2). This will be done by application of the lemma together with the fact that the convergence of the polynomials to $f(z)$ is continuous in $G$. Let $\epsilon$ be an arbitrarily small positive number. Let $H$ be any subregion of $G$ such that the closed region $\bar{H}$ lies interior to $G$ and also such that

$$
\iint_{G-H}|f(z)|^{p} d S<\epsilon .
$$


The polynomials $\left\{p_{n}(z)\right\}$ established in the preceding paragraph are such that from a certain value of $n$ on, say for $n_{0} \leqq n$, the following three conditions are simultaneously satisfied:

$$
\begin{aligned}
& \left.\left|\iint_{G}\right| p_{n}(z)\right|^{p} d S-\iint_{G}|f(z)|^{p} d S \mid<\epsilon, \\
& \left|p_{n}(z)-f(z)\right|<\epsilon, \\
& \left.\left|\iint_{H}\right| p_{n}(z)\right|^{p} d S-\iint_{H}|f(z)|^{p} d S \mid<\epsilon .
\end{aligned} \quad(z \text { in } \bar{H}),
$$

From (6), (7), and (9) it follows that

$$
\iint_{G-H}\left|p_{n}(z)\right|^{p} d S<3 \epsilon, \quad\left(n_{0} \leqq n\right) .
$$

Let $A=\iint_{G} d S$. Then

$$
\begin{aligned}
\iint_{G}\left|f(z)-p_{n}(z)\right|^{p} d S= & \iint_{H}\left|f(z)-p_{n}(z)\right|^{p} d S \\
& +\iint_{G-H}\left|f(z)-p_{n}(z)\right|^{p} d S \\
\leqq & \epsilon^{p}+2^{p}(4 \epsilon),
\end{aligned}
$$

where the second integral on the right has been appraised by the lemma. The quantity $m(\epsilon)=A \epsilon^{p}+2^{p}(4 \epsilon)$ is clearly a function of $\epsilon$ which approaches zero as limit along with $\epsilon$.Consequently the polynomials $\left\{p_{n}(z)\right\}$ which converge to $f(z)$ continuously in $G$ and for which (5) holds must also satisfy (2).

4. A Comparison of the Two Theorems. It may not be without interest to compare these two theorems with each other and with Runge's* fundamental theorem on polynomial approximation, at least in the case of a simple example. For our example we take $p=1, f(z)=z$, and for $G$ the interior of the unit circle, $|z|<1$. The polynomials $p_{n}(z)=n z^{n}+z,(n=1,2, \cdots)$, converge to $f(z)$ continuously in $G$, but they do not satisfy (2), for p. 578 .

* See, for instance, Osgood, Lehrbuch der Funktionentheorie, vol. 1, 1928, 


$$
\begin{aligned}
\lim _{n \rightarrow \infty} \iint_{G}\left|f(z)-p_{n}(z)\right| d S & =\lim _{n \rightarrow \infty} \int_{0}^{2 \pi} \int_{0}^{1} n r^{n+1} d r d \theta \\
\quad\left(z=r e^{i \theta}\right), & \\
& =\lim _{n \rightarrow \infty} \frac{2 \pi n}{n+2}=2 \pi .
\end{aligned}
$$

On the the other hand the polynomials $P_{n}(z)=z^{n}+z$ not only converge to $f(z)$ continuously in $G$ but also satisfy (2), as may be readily verified. They do not, however, satisfy (1), for bound $\left[\left|P_{n}(z)\right|, z\right.$ in $\left.G\right]=2$ for every $n$. Finally the polynomials $\pi_{n}(z)=\left(z^{n} / n\right)+z$ converge to $f(z)$ continuously in $G$ and at the same time satisfy (1).

5. A Remark on Theorem 1. Theorem 1 can not be extended to the most general finite simply connected region. Essentially the same remark was made without amplification by Carleman in the paper cited. The writer feels, however, that the following detailed justification of the remark is not unworthy of consideration. A region such that Theorem 1 can not hold for an arbitrary function $f(z)$ analytic and bounded therein is any finite simply connected region $R$ such that the complete boundary of $R$ is not the boundary of an infinite region, as for instance a circle from which one radius has been removed or again a region consisting of the points of a strip which lies inside a circle, is closed at one end, and winds around infinitely often, approaching as limit every point of the circle. Let $B$ denote that portion of the boundary of $R$ which is the boundary of an infinite region. All the points of the plane which can be joined to a fixed point of $R$ by a Jordan arc containing no point of $B$ form a region $S$ which contains not only all the points of $R$ but boundary points of $R$ as well. Let $f(z)$ be a function which maps $R$ conformally onto the interior of the unit circle. If polynomials $\left\{p_{n}(z)\right\}$ existed which converged to $f(z)$ in $R$ and for which (1) held, it would follow from the continuity of these polynomials in the closed region $\bar{R}$ that the $\left\{p_{n}(z)\right\}$ would be uniformly bounded in $S$ and hence form a normal family in $S . *$ A subsequence of these polynomials could then be found which would

* See Montel, Lȩons sur les Familles Normales de Fonctions Analytiques, 1927, pp. 21-33. 
converge to a function $\phi(z)$ analytic in $S$ and such that $|\phi(z)|<1$ for $z$ in $S$. Since the original sequence $\left\{p_{n}(z)\right\}$ converges by hypothesis in $R$, the limit function of the subsequence would necessarily agree with $f(z)$ in $R$. But $\phi(z)$ could not be identical with $f(z)$ in $R$, for then $\phi(z)$ would have to equal unity at interior points of $S$, namely, at the boundary points of $R$ which lie interior to $S$.

It would appear from this remark that Theorem 1 (and Theorem 2 as well) might admit of extension to an arbitrary finite simply connected region whose complete boundary is also the boundary of an infinite region, and that a modification of the methods used in the proofs of the present paper would serve to establish such an extension. The writer hopes to answer this question in a later paper.

Union College,

Schenectady, N. Y.

\section{NOTE ON A SIMPLE TYPE OF ALGEBRA IN WHICH THE CANCELLATION LAW OF ADDITION DOES NOT HOLD}

\section{BY H. S. VANDIVER}

1. Introduction. I do not imagine that the algebraic system considered in this note can be new, but if it has been overlooked this has probably happened because of its simplicity. However, we shall be most interested here in examining the connection of the system with the foundations of ordinary algebra. As we shall see, the symbols employed have most of the properties of rational integers, the principal exceptions being that they are finite in number and from

$$
a+b=a+c
$$

we cannot infer $b=c$ in general.*

2. Description of the System. Suppose we introduce the natural numbers $1,2,3, \cdots$, employing for their use Peano's system

* In a system in which we may always infer $b=c$ under the condition given we shall say the cancellation law holds. 